\title{
On the numerical solution of fractional differential equations with cubic nonlinearity via matching polynomial of complete graph
}

\author{
ÖMÜR KIVANÇ KÜRKÇÜ̈ ${ }^{1, *}$, ERSIN ASLAN ${ }^{2}$ and MEHMET SEZER ${ }^{3}$ \\ ${ }^{1}$ Department of Mathematics, İzmir University of Economics, 35330 Izmir, Turkey \\ ${ }^{2}$ Department of Software Engineering, Manisa Celal Bayar University, 45400 Manisa, Turkey \\ ${ }^{3}$ Department of Mathematics, Manisa Celal Bayar University, 45140 Manisa, Turkey \\ e-mail: omurkivanc@outlook.com; ersin.aslan@cbu.edu.tr; mehmet.sezer@cbu.edu.tr
}

MS received 26 April 2019; revised 27 August 2019; accepted 24 September 2019

\begin{abstract}
This study deals with a generalized form of fractional differential equations with cubic nonlinearity, employing a matrix-collocation method dependent on the matching polynomial of complete graph. The method presents a simple and efficient algorithmic infrastructure, which contains a unified matrix expansion of fractional-order derivatives and a general matrix relation for cubic nonlinearity. The method also performs a sustainable approximation for high value of computation limit, thanks to the inclusion of the matching polynomial in matrix system. Using the residual function, the convergence and error estimation are investigated via the second mean value theorem having a weight function. In comparison with the existing results, highly accurate results are obtained. Moreover, the oscillatory solutions of some model problems arising in several applied sciences are simulated. It is verified that the proposed method is reliable, efficient and productive.
\end{abstract}

Keywords. Fractional differential equations; matrix-collocation method; convergence analysis; Laplace-Padé method.

\section{Introduction}

In the past 40 years, fractional differential equations (FDEs) have received much attention in describing many physical phenomena, which arise mainly in applied sciences, such as mathematics, engineering, physical processes, signal processing, anomalous diffusion, biology, chemistry, fluid mechanics and electromagnetics [1-10]. As these phenomena are evolved in the developing world, FDEs start to exactly govern them instead of integer-order differential equations. In addition, we draw attention to the fact that FDEs including nonlinear terms have recently been of importance for modelling real world problems in physical sense. However, their physical behaviours cannot be entirely estimated since finding their exact solutions is hard. Furthermore, nonlinear FDEs have frequently no exact solutions. Hence, a great amount of effort has been devoted to finding the numerical solutions. Especially, there has been a remarkable increment of development of numerical methods for solving FDEs in the last decade. From this point of view, so far, Kürkçü et al [11, 12] have introduced and employed a novel graph-operational matrix method for multidelay linear FDEs and quintic nonlinear FDEs. Gülsu et al [4] have applied the Taylor polynomial method to solve fractional Ricatti equation. Yüzbaşı [13]

*For correspondence

Published online: 30 November 2019 has proposed the Bernstein polynomial method for fractional Ricatti type differential equations. Saeed [14] has established the CAS Picard method for numerical solutions of the nonlinear FDEs. Yarmohammadi [15] have presented the spectral iterative method and its convergence analysis for treating nonlinear FDEs. Rehman and Khan [16] have employed the Legendre wavelet method (LWM) to obtain the approximate solutions of FDEs.

On the other hand, it is known that the matrix-collocation methods based on specific polynomials, such as Lucas polynomial [17], Taylor polynomial [18, 19] and Dickson polynomials [20], provide precise numerical solutions of integer-order differential equations of several types. Also, in [11], we use a fundamental structure of linear FDEs in the proposed method, which contains linear matrix relations. Further, we observe that this method immediately yields clear and efficient approximate solutions. Here, in this study, we aim to develop further matrix relations of the method, which treat both linear and nonlinear terms of FDEs. This developed method has an advantage over the existing methods since it makes use of the matching polynomial of complete graph, a unified matrix expansion of fractional-order derivative and a general matrix relation for cubic nonlinearity. Thus, we can deal with a wide range of FDEs with cubic nonlinearity under a unique formulation. A unique computer program of the method can also be easily devised and run 
on any mathematical software, thanks to its simple and efficient algorithmic infrastructure.

This study is organized as follows. Section 2 gives some basics of fractional calculus and matching polynomial, which are required to construct the proposed method. Section 3 reveals the construction of the methodology. Section 4 is on the oscillatory behaviour of the obtained solutions via the present and Laplace-Padé methods. Section 5 scrutinizes the convergence analysis and error estimation with the aid of the second mean value theorem for integrals. Section 6 presents stiff numerical problems and their obtained results. Section 7 interprets the obtained results via the proposed method and its validity according to the investigation of the present results found in section 6 .

A generalized form of FDE with cubic nonlinearity is

$$
\begin{aligned}
& \sum_{0 \leq \alpha \leq m_{1}} P_{\alpha} y^{(\alpha)}(t) \\
& \quad+\sum_{0 \leq r_{1} \leq m_{2}} \sum_{0 \leq r_{2} \leq r_{1}} \sum_{0 \leq r_{3} \leq r_{2}} Q_{r_{1}, r_{2}, r_{3}}\left[y^{\left(r_{1}\right)} y^{\left(r_{2}\right)} y^{\left(r_{3}\right)}\right](t) \\
& =g(t),
\end{aligned}
$$

subject to the mixed conditions

$$
\sum_{k=0}^{m-1}\left[a_{i k} y^{(k)}(a)+b_{i k} y^{(k)}(b)\right]=\psi_{i}, m=\max \left\{m_{1}, m_{2}\right\},
$$

where $t \in[a, b], y(t)$ and $g(t)$ are defined on $[a, b]$; $\left\{\alpha, r_{1}, r_{2}, r_{3}\right\}$ are integer- or fractional-order derivatives; $P_{\alpha}, Q_{r_{1}, r_{2}, r_{3}}$ and $a_{i k}, b_{i k}(i=0,1, \ldots, m-1)$ are proper constants; $m_{1}, m_{2} \in \mathbb{Z}^{0+}$.

The matching polynomial solution of Eq. (1) that we want to seek is of the form (see $[11,12]$ )

$$
y(t) \cong y_{N}(t)=\sum_{n=0}^{N} y_{n} M_{n}\left(K_{n}, t\right)
$$

where $y_{n}$ are unknown coefficients and $M\left(K_{n}, t\right)$ is the matching polynomial of complete graph $K_{n}$.

\section{Some basics of fractional calculus and matching polynomial}

\subsection{Fractional calculus}

In this section we briefly mention the fundamental basics of fractional calculus, which are of importance for the proposed method. Let $f(t)$ be a continuous function on

$$
I=\{[a, b]: a, b \in \mathbb{R}\} .
$$

$D$ is a differential operator and $\Gamma(\alpha)$ is the Euler gamma function. We can now state the following definitions.

Definition 2.1 $[2,8]$ Suppose that $\alpha>0$ and $t>a$. Then the fractional Riemann-Liouville integral of $f(t)$ is defined to be

$$
J_{a}^{\alpha} f(t)=\frac{1}{\Gamma(\alpha)} \int_{a}^{t}(t-s)^{\alpha-1} f(s) d s
$$

where $\alpha, t \in \mathbb{R}$ and $J_{a}^{\alpha}$ is a fractional integral operator.

Definition 2.2 $[2,8]$ Suppose that $t>a$ and $\alpha, t \in \mathbb{R}$. Then the fractional Riemann-Liouville derivative of order $\alpha$ is defined to be

$$
\begin{aligned}
& { }_{t}^{R L} D_{a}^{\alpha} f(t)=\frac{1}{\Gamma(\lceil\alpha\rceil-\alpha)} D^{\lceil\alpha\rceil} \int_{a}^{t}(t-s)^{\lceil\alpha\rceil-\alpha-1} f(s) d s, \\
& \lceil\alpha\rceil-1<\alpha<\lceil\alpha\rceil
\end{aligned}
$$
and $f^{(\alpha)}(t)$ stands for $\alpha \in \mathbb{N}$.

Definition 2.3 $[1,2,8]$ Suppose that $t>a$ and $\alpha, t \in \mathbb{R}$. Then the fractional Caputo derivative of order $\alpha$ is defined to be

$$
\begin{gathered}
{ }_{t}^{C} D_{a}^{\alpha} f(t)=\frac{1}{\Gamma(\lceil\alpha\rceil-\alpha)} \int_{a}^{t}(t-s)^{\lceil\alpha\rceil-\alpha-1} f^{(\lceil\alpha\rceil)}(s) d s, \\
\lceil\alpha\rceil-1<\alpha<\lceil\alpha\rceil,
\end{gathered}
$$

and $f^{(\alpha)}(t)$ stands for $\alpha \in \mathbb{N}$. Here, ${ }_{t}^{C} D_{a}^{\alpha}$ is the fractional Caputo derivative operator.

In addition to these definitions, new formulations of the fractional derivative types have recently been introduced in terms of their kernel properties. For example, fractional Caputo-Fabrizio derivative [21-23] and Atangana-Baleanu derivative [24] formulations contain nonlocal and non-singular kernels. Both are described as follows:

Definition 2.4 [21-23] Suppose that $t \geq 0$. Then the fractional Caputo-Fabrizio derivative of order $\alpha$ is defined to be

$$
{ }_{t}^{C F} D_{0}^{\alpha} f(t)=\frac{1}{1-\alpha} \int_{0}^{t} \exp \left(-\frac{\alpha(t-s)}{1-\alpha}\right) f^{\prime}(s) d s, 0<\alpha<1 .
$$

When $m \geq 1$ and $\alpha \in[0,1],{ }_{t}^{C F} D_{0}^{m+\alpha} f(t)$ is of the form [21-23]

$$
{ }_{t}^{C F} D_{0}^{\alpha+m} f(t)={ }_{t}^{C F} D_{0}^{\alpha}\left({ }_{t}^{C} D_{0}^{m} f(t)\right) .
$$

Definition 2.5 [24] Suppose that $f \in H^{1}(a, b), b>a$. Then the fractional Atangana-Baleanu derivative of order $\alpha$ is defined to be 


$$
{ }_{t}^{A B C} D_{b}^{\alpha} f(t)=\frac{N(\alpha)}{1-\alpha} \int_{b}^{t} E_{\alpha}\left[-\alpha \frac{(t-s)^{\alpha}}{1-\alpha}\right] f^{\prime}(s) d s, 0 \leq \alpha \leq 1
$$

where $N(\alpha)$ is a normalization function with $N(0)=$ $N(1)=1$ and $E_{\alpha}[\cdot]$ is the Mittag-Leffler function.

More recently, Doungmo Goufo [25] used the fractional Caputo-Fabrizio derivative for treating the Korteweg-de Vries-Bergers equation and also Doungmo Goufo [26] investigated the chaotic processes according to a two-parameter derivative having non-local and non-singular kernel. For specific properties and applications of fractional derivative formulations with non-singular and non-local kernels, the reader can refer to [21-26]. It can also be easily concluded from these formulations that the fractional derivative operator provides both integer- and fractionalorder derivatives. In this study, we use ${ }_{t}^{C} D_{a}^{\alpha} y(t)$ as $y^{(\alpha)}(t)(a=0)$ in the definition of the fractional Caputo derivative.

\subsection{Matching polynomial}

In 1972, Heilman and Lieb [27] first used a polynomial for the theory of monomer-dimer systems without determining its specific name. In 1979, Farrell [28] denominated it as the matching polynomial, which is made up of collecting $k$ matching numbers of independent edges in a graph. So far, some authors have used it in different names, such as acyclic [29] and reference [30]. Let $G_{n}$ be a simple graph with $n$ vertices and $m$ edges [31]; then the matching polynomial of $G_{n}$ is generally defined to be (see [28-30, 32, 33])

$$
M_{n}\left(G_{n}, t\right)=\sum_{k=0}^{m}(-1)^{k} p\left(G_{n}, k\right) t^{n-2 k}
$$

where $p\left(G_{n}, 0\right)=1$ and $p\left(G_{n}, k\right)$ is the matching number.

The matching polynomial of complete graph denoted by $K_{n}$ is of the explicit form (see [29, 32])

$$
M_{n}\left(K_{n}, t\right)=\sum_{k=0}^{\lfloor n / 2\rfloor}(-1)^{k} \frac{(2 k) !}{2^{k} k !}\left(\begin{array}{c}
n \\
2 k
\end{array}\right) t^{n-2 k},
$$

and its recurrence relation is

$$
M_{n}\left(K_{n}, t\right)=t M_{n}\left(K_{n-1}, t\right)-(n-1) M_{n}\left(K_{n-2}, t\right),
$$

which are equivalent to the modified Hermite polynomials (see [29, 32]). The readers can also see the other matching polynomials emerging from path, wheel, cycle and star graphs [29, 32, 34].

On the other hand, up to now, Aihara [30] has used the reference (matching) polynomial for monocyclic conjugated system. Godsil and Gutman [33] have presented the properties of the matching polynomial. Hosoya [35] has proposed the mathematical properties of the matching and some other polynomials in chemistry. Ghosh et al [36] have studied the matching polynomial coefficients and Hosoya indices for the linear and cylindrical polygraphs. Araujo et al [37] have investigated the correlation of the matching polynomial and the hypergeometric functions by establishing the general form of the matching polynomial.

Here, the reason why we take the matching polynomial of $K_{n}$ is that $K_{n}$ is a strong-structured graph, which cannot be easily scattered as defined in the vulnerability theory $[31,38,39]$ because all its vertices are connected to each other via edges. Figure 1 shows this durability for $K_{10}$. It is thus important to state that the matching polynomial of $K_{n}$ has a stable structure. However, this situation can be varied according to the afore-mentioned graph classes.

\section{Procedure of method}

In this section, the matrix relations of the matching polynomial, the fractional-order derivatives in Caputo sense and nonlinear terms are constructed. They form a basis of the method. At first, the matrix relation of Eq. (3) is given as (see $[11,12])$

$$
y(t)=\boldsymbol{M}\left(K_{n}, t\right) \boldsymbol{Y}=\boldsymbol{X}(t) \boldsymbol{K} \boldsymbol{Y},
$$

where

$$
\begin{aligned}
\boldsymbol{M}\left(K_{n}, t\right) & =\left[\begin{array}{lllll}
M_{0}\left(K_{0}, t\right) & M_{1}\left(K_{1}, t\right) & \ldots & M_{N}\left(K_{N}, t\right)
\end{array}\right], \\
\boldsymbol{X}(t) & =\left[\begin{array}{lllll}
1 & t & t^{2} & \cdots & t^{N}
\end{array}\right], \\
\boldsymbol{Y} & =\left[\begin{array}{lllll}
y_{0} & y_{1} & \ldots & y_{N}
\end{array}\right]^{T},
\end{aligned}
$$

and $\boldsymbol{K}^{T}$ is a lower triangular matrix, which can be easily obtained by taking the coefficients of the matching polynomial of complete graph $K_{n}[11,12]$.

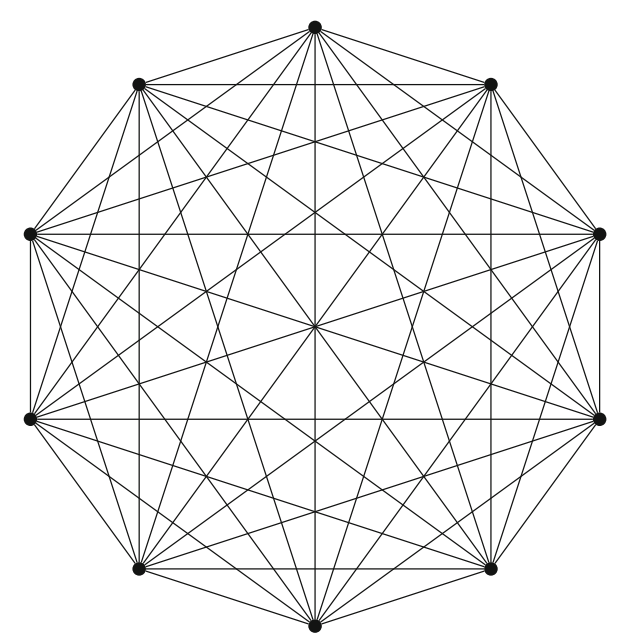

Figure 1. A complete graph $K_{10}$ with 10 vertices. 
Let us now state the fractional derivative of the matrix relation (4) by supposing that $\alpha \geq 0$ as (see [11, 12])

$$
y^{(\alpha)}(t)=\boldsymbol{M}^{(\alpha)}\left(K_{n}, t\right) \boldsymbol{Y}=\boldsymbol{X}^{(\alpha)}(t) \boldsymbol{K} \boldsymbol{Y}
$$

where

$$
\begin{aligned}
& \boldsymbol{M}^{(\alpha)}\left(K_{n}, t\right) \\
& \quad=\left[{ }_{t}^{C} D_{0}^{\alpha}\left(M_{0}\left(K_{0}, t\right)\right) \quad \ldots \quad{ }_{t}^{C} D_{0}^{\alpha}\left(M_{N}\left(K_{N}, t\right)\right)\right],
\end{aligned}
$$

and

$\boldsymbol{X}^{(\alpha)}(t)=\left[\begin{array}{lllll}{ }_{t}^{C} D_{0}^{\alpha}(1) & { }^{C} D_{0}^{\alpha}(t) & { }_{t}^{C} D_{0}^{\alpha}\left(t^{2}\right) & \cdots & { }_{t}^{C} D_{0}^{\alpha}\left(t^{N}\right)\end{array}\right]$.

By Eq. (5) and using the collocation points $t_{i}=a+i(b-a) / N$, the matrix relation of linear part $L[y(t)]$ of Eq. (1) turns out to be

$$
\left[L\left[y\left(t_{i}\right)\right]\right]=\boldsymbol{P}_{\alpha} \boldsymbol{X}^{(\alpha)}\left(t_{i}\right) \boldsymbol{K} \boldsymbol{Y},
$$

where $0 \leq \alpha \leq m_{1}$.

Let us now construct the matrix relation of nonlinear part $N[y(t)]$ of Eq. (1). For cubic nonlinearity, using again the collocation points, we can state

$$
\left[N\left[y\left(t_{i}\right)\right]=\boldsymbol{Q}_{r_{1}, r_{2}, r_{3}} \boldsymbol{X}^{\left(r_{1}\right)}\left(t_{i}\right) \boldsymbol{K} \overline{\boldsymbol{X}^{\left(r_{2}\right)}\left(t_{i}\right)} \overline{\boldsymbol{K}} \overline{\overline{\boldsymbol{X}^{\left(r_{3}\right)}\left(t_{i}\right)}} \overline{\overline{\boldsymbol{K}}} \overline{\overline{\boldsymbol{Y}}}\right.
$$

where $0 \leq r_{1} \leq m_{2}, 0 \leq r_{2} \leq r_{1}$ and $0 \leq r_{3} \leq r_{2}$.

Especially for $m_{2}=0$ (hence $r_{1}=0$ ),

$$
\left[Q_{0,0,0} y^{3}\left(t_{i}\right)\right]=\boldsymbol{Q}_{0,0,0} \boldsymbol{X}\left(t_{i}\right) \boldsymbol{K} \overline{\boldsymbol{X}\left(t_{i}\right)} \overline{\boldsymbol{K}} \overline{\overline{\boldsymbol{X}\left(t_{i}\right)}} \overline{\overline{\boldsymbol{K}}} \overline{\overline{\boldsymbol{Y}}} .
$$

Notice that the matrix relation (7) can be easily reduced to the quadratic nonlinearity:

$$
\left[Q_{r_{1}, r_{2}} y^{\left(r_{1}\right)}\left(t_{i}\right) y^{\left(r_{2}\right)}\left(t_{i}\right)\right]=\boldsymbol{Q}_{r_{1}, r_{2}} \boldsymbol{X}^{\left(r_{1}\right)}\left(t_{i}\right) \boldsymbol{K} \overline{\boldsymbol{X}^{\left(r_{2}\right)}\left(t_{i}\right)} \overline{\boldsymbol{K}} \overline{\boldsymbol{Y}}
$$

where $0 \leq r_{1} \leq m_{2}$ and $0 \leq r_{2} \leq r_{1}$.

Gathering and simplifying the matrix relations (6) and (7) (or (8)), we get

$$
\begin{aligned}
& \boldsymbol{P}_{\alpha} \boldsymbol{X}^{(\alpha)} \boldsymbol{K} \boldsymbol{Y}+\boldsymbol{Q}_{r_{1}, r_{2}, r_{3}} \boldsymbol{X}^{\left(r_{1}\right)} \boldsymbol{K} \overline{\boldsymbol{X}^{\left(r_{2}\right)}} \overline{\boldsymbol{K}} \overline{\overline{\boldsymbol{X}^{\left(r_{3}\right)}}} \overline{\overline{\boldsymbol{K}}} \overline{\overline{\boldsymbol{Y}}} \\
& \quad=\boldsymbol{G}
\end{aligned}
$$

where $0 \leq \alpha \leq m_{1}, 0 \leq r_{1} \leq m_{2}, 0 \leq r_{2} \leq r_{1}$ and $0 \leq r_{3} \leq r_{2}$.

It follows from the matrix relation (9) that

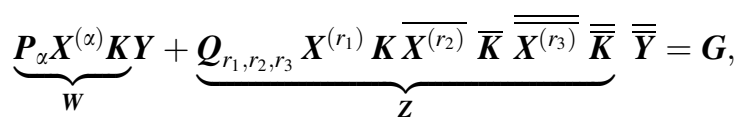

where

$$
\boldsymbol{P}_{\alpha}=\operatorname{diag}\left[P_{\alpha}\right]_{(N+1) \times(N+1)},
$$

$\boldsymbol{Q}_{r_{1}, r_{2}, r_{3}}=\operatorname{diag}\left[Q_{r_{1}, r_{2}, r_{3}}\right]_{(N+1) \times(N+1)}$,

$\boldsymbol{X}^{(\alpha)}=\left[\begin{array}{c}\boldsymbol{X}^{(\alpha)}\left(t_{0}\right) \\ \boldsymbol{X}^{(\alpha)}\left(t_{1}\right) \\ \vdots \\ \boldsymbol{X}^{(\alpha)}\left(t_{N}\right)\end{array}\right]$

$=\left[\begin{array}{cccc}{ }_{t}^{C} D_{0}^{\alpha}(1) & { }_{t}^{C} D_{0}^{\alpha}\left(t_{0}\right) & \cdots & { }_{t}^{C} D_{0}^{\alpha}\left(t_{0}^{N}\right) \\ { }_{t}^{C} D_{0}^{\alpha}(1) & { }_{t}^{C} D_{0}^{\alpha}\left(t_{1}\right) & \cdots & { }_{t}^{C} D_{0}^{\alpha}\left(t_{1}^{N}\right) \\ \vdots & \vdots & \ddots & \vdots \\ { }_{t}^{C} D_{0}^{\alpha}(1) & { }_{t}^{C} D_{0}^{\alpha}\left(t_{N}\right) & \cdots & { }_{t}^{C} D_{0}^{\alpha}\left(t_{N}^{N}\right)\end{array}\right]$,

$\overline{\boldsymbol{X}^{\left(r_{2}\right)}}=\left[\begin{array}{cccc}\boldsymbol{X}^{\left(r_{2}\right)}\left(t_{0}\right) & 0 & \cdots & 0 \\ 0 & \boldsymbol{X}^{\left(r_{2}\right)}\left(t_{1}\right) & \cdots & 0 \\ \vdots & \vdots & \ddots & \vdots \\ 0 & 0 & 0 & \boldsymbol{X}^{\left(r_{2}\right)}\left(t_{N}\right)\end{array}\right]_{(N+1) \times(N+1)^{2}}$,

$\overline{\overline{\boldsymbol{X}^{\left(r_{3}\right)}}}=\operatorname{diag}\left[\overline{\boldsymbol{X}^{\left(r_{3}\right)}}\right]_{(N+1)^{2} \times(N+1)^{3}}$,

$\overline{\boldsymbol{K}}=\operatorname{diag}[\overline{\boldsymbol{K}}]_{(N+1)^{2} \times(N+1)^{2}}, \overline{\overline{\boldsymbol{K}}}=\operatorname{diag}[\overline{\boldsymbol{K}}]_{(N+1)^{3} \times(N+1)^{3}}$,

$\overline{\boldsymbol{Y}}=\left[\begin{array}{llll}y_{0} \boldsymbol{Y} & y_{1} \boldsymbol{Y} & \cdots & y_{N} \boldsymbol{Y}\end{array}\right]^{T}$,

$\overline{\overline{\boldsymbol{Y}}}=\left[\begin{array}{llll}y_{0} \overline{\boldsymbol{Y}} & y_{1} \overline{\boldsymbol{Y}} & \ldots & y_{N} \overline{\boldsymbol{Y}}\end{array}\right]^{T}$

and

$$
\boldsymbol{G}=\left[\begin{array}{llll}
g\left(t_{0}\right) & g\left(t_{1}\right) & \cdots & g\left(t_{N}\right)
\end{array}\right]^{T} .
$$

On the other hand, using Eq. (5), we present the matrix relation of the mixed conditions (2) as the following:

$$
\sum_{k=0}^{m-1}\left[a_{i k} \boldsymbol{M}^{(k)}\left(K_{n}, a\right)+b_{i k} \boldsymbol{M}^{(k)}\left(K_{n}, b\right)\right] \boldsymbol{Y}=\psi_{i},
$$

where $i=0,1, \ldots, m-1$.

By the matrix equation (10), we can construct the method of solution as

$$
\boldsymbol{W Y}+\boldsymbol{Z} \overline{\overline{\boldsymbol{Y}}}=\boldsymbol{G} \text { or }[\boldsymbol{W} ; \boldsymbol{Z}: \boldsymbol{G}] .
$$

In view of the matrix relation (11), we can get

$$
\boldsymbol{U}_{i} \boldsymbol{Y}=\psi_{i} \Rightarrow\left[\boldsymbol{U}_{i}: \psi_{i}\right], i=0,1, \ldots m-1
$$

where

$$
\boldsymbol{U}_{i} \equiv\left[\begin{array}{llll}
u_{i 0} & u_{i 1} & \cdots & u_{i N}
\end{array}\right] .
$$

Replacing the conditional matrix (13) by any $m$-th row(s) of the matrix equation (12), we then obtain the augmented matrix $[\widetilde{\boldsymbol{W}} ; \widetilde{\boldsymbol{Z}}: \widetilde{\boldsymbol{G}}]$. Then, the augmented matrix can be solved only if its rank yields $N+1$. Therefore, by substituting the resulting matrix $\boldsymbol{Y}$ into the solution form (3), the matching polynomial solution is thereby found. 


\section{Estimation of oscillatory behaviour via matrix- collocation and Laplace-Padé methodology}

Since it is often hard to numerically determine the oscillatory behaviour of nonlinear phenomena on long time interval, the authors in $[40,41]$ merged their methods with Laplace-Padé method. Thereby, they reached the oscillatory behaviour of the solutions of the coupled nonlinear partial differential equations and the non-linear oscillators. For this motivation, we combine the present method with Laplace-Padé method described in [40, 41]. The algorithmic procedure of matrix-collocation and Laplace-Padé methodology can be described as follows.

Step 1: $G(s) \leftarrow L\left\{y_{N}(t)\right\}=\int_{0}^{\infty} y_{N}(t) e^{-s t} d t$, where $L\{\cdot\}$ is Laplace transform and $y_{N}(t)$ is the matching polynomial solution,

Step 2: $\quad G\left(\frac{1}{t}\right) \underset{s \rightarrow 1 / t}{\leftarrow} G(s)$

Step 3: $\quad H(s) \underset{t \rightarrow 1 / s}{\leftarrow} H\left(\frac{1}{t}\right) \leftarrow P\left[G\left(\frac{1}{t}\right)\right],(K, L$ : numerator and denominator degree, respectively $(K, L<M))$, $(P[$.$] is the well-known Padé approximant),$

Step 4: $\quad y_{P, N}(t) \leftarrow L^{-1}\{H(s)\}$,

where $y_{P, N}(t)$ is the Padé-matching polynomial solution and $L^{-1}\{$.$\} is inverse Laplace transform. Thus, we can estimate$ the oscillatory behaviour of the solutions on long time interval.

\section{Convergence analysis and error estimation based on the second mean value theorem}

In this section, we deal with the second mean value theorem for integrals, which dates back to some calculus studies by Hobson [42] and Dixon [43] in 20th century, to construct the convergence analysis and error estimation of the

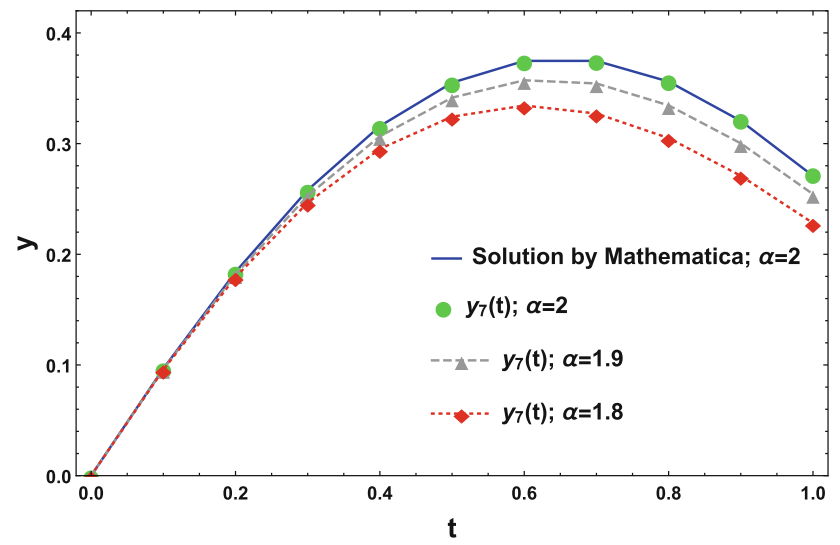

Figure 2. Comparison of the solutions for Problem 6.1 with high damping effect $\mu=0.5$ and $T=1$. proposed method. First, let us now state the second mean value theorem as a lemma.

Lemma 5.1 $[42,43]$ Let $f(t):[a, b] \mapsto \mathbb{R}^{+}$be an integrable function and $\omega(t):[a, b] \mapsto \mathbb{R}^{+}$be an integrable positive function; then

$$
\int_{a}^{b} f(t) \omega(t) d t=f(c) \int_{a}^{b} \omega(t) d t
$$

such that $c \in[a, b]$ and $f(c)$ is called an average value of $f(t)$ with respect to the weight function $\omega(t)$ on $[a, b]$. For $\omega(t)=1$

$$
\int_{a}^{b} f(t) \omega(t) d t=f(c)(b-a),
$$

which is called the first mean value theorem for integrals.

Before constructing the convergence analysis and error estimation of the method, let us state the residual function, which is required for our analysis. After inserting the approximate solution $y_{N}(t)$ into Eq. (1), the residual function $R_{N}(t)$ is obtained as

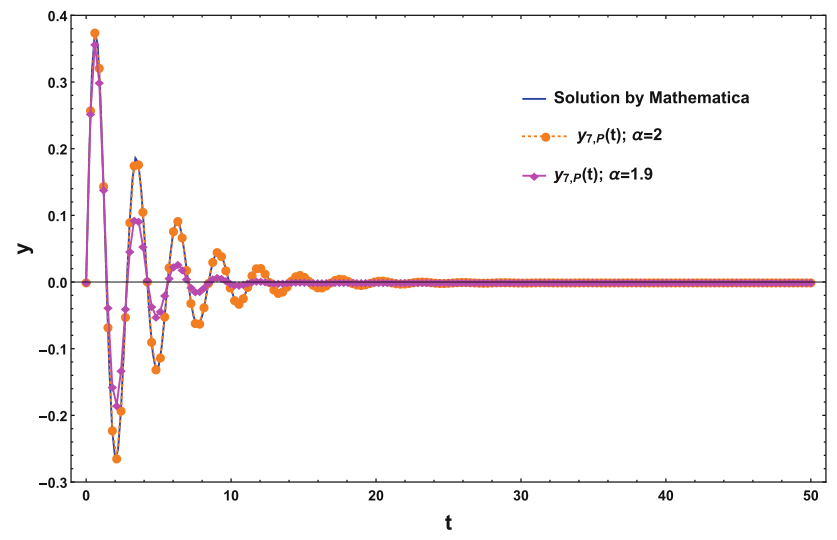

Figure 3. Oscillatory behaviour of the solutions for Problem 6.1 with high damping effect $\mu=0.5$ and $T=50$.

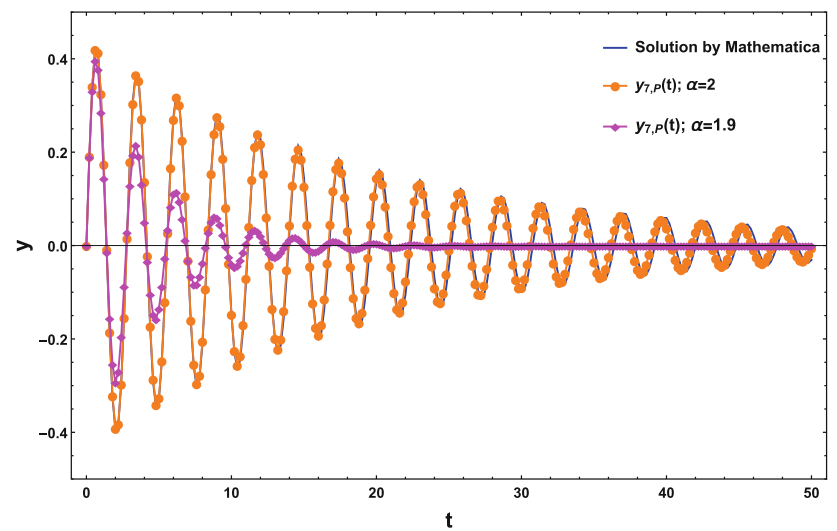

Figure 4. Oscillatory behaviour of the solutions for Problem 6.1 with low damping effect $\mu=0.1$ and $T=50$. 


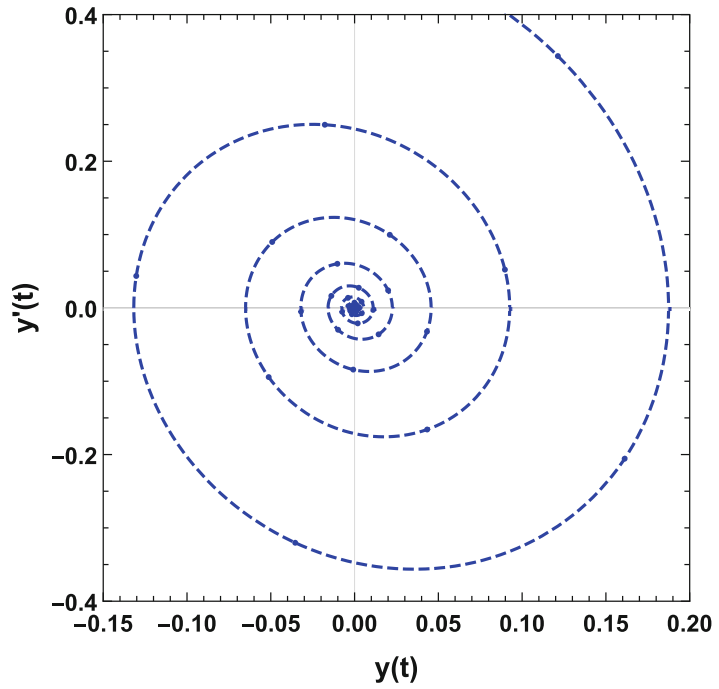

(a) Solution by Mathematica; $\alpha=2$.

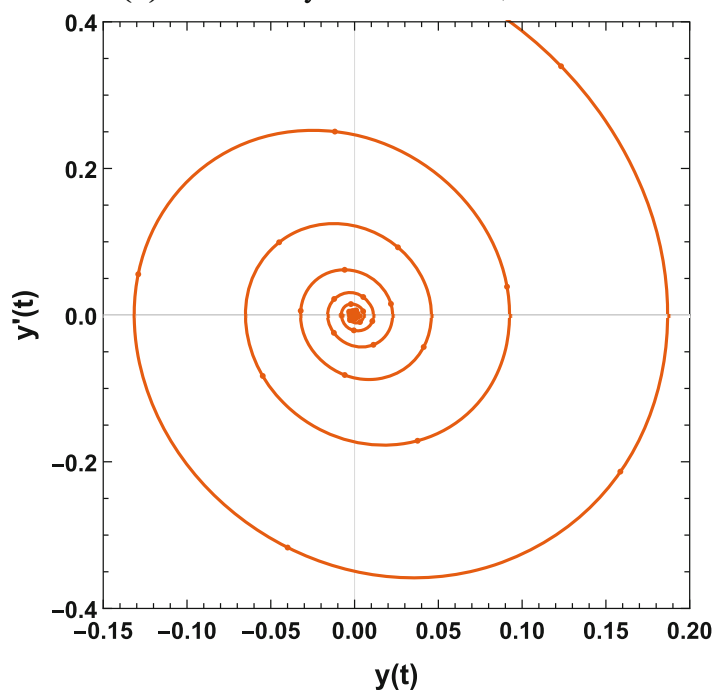

(b) $y_{7, P}(t) ; \alpha=2$.

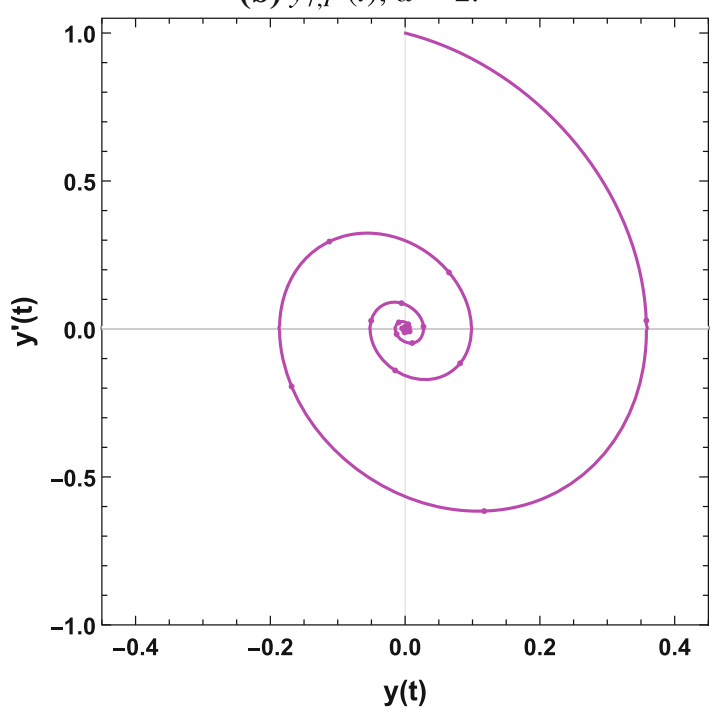

(c) $y_{7, P}(t) ; \alpha=1.9$
4Figure 5. Phase plane behaviour of the solutions for Problem 6.1 with high damping effect $\mu=0.5$ and $T=50$.

$$
\begin{aligned}
R_{N}(t)= & \sum_{0 \leq \alpha \leq m_{1}} P_{\alpha} y_{N}^{(\alpha)}(t) \\
& +\sum_{0 \leq r_{1} \leq m_{2}} \sum_{0 \leq r_{2} \leq r_{1}} \sum_{0 \leq r_{3} \leq r_{2}} Q_{r_{1}, r_{2}, r_{3}} \\
& {\left[y_{N}^{\left(r_{1}\right)} y_{N}^{\left(r_{2}\right)} y_{N}^{\left(r_{3}\right)}\right](t)-g(t) . }
\end{aligned}
$$

By Lemma 5.1 and Eq. (14), we can now construct the convergence analysis, which investigates the precision of the method with respect to $N$.

Theorem 5.1 Let $R_{N}(t)$ be an integrable function on $[a, b]$ and $\omega(\lambda t)(\lambda \in \mathbb{R})$ be a parameterized positive weight function of $R_{N}(t)$; then

$$
\hat{R}_{N}=\frac{\left|\int_{a}^{b} R_{N}(t) \omega(\lambda t) d t\right|}{\int_{a}^{b} \omega(\lambda t) d t}
$$

determines the precision of the method as the upper bound error with respect to the computation limit $N$ and $\omega(\lambda t)$.

Proof We know the property of inequality for integrals, so we can write

$$
\left|\int_{a}^{b} R_{N}(t) \omega(\lambda t) d t\right| \leq \int_{a}^{b}\left|R_{N}(t) \omega(\lambda t)\right| d t .
$$

Since the weight function $\omega(\lambda t)$ is a positive function, it yields

$$
\left|\int_{a}^{b} R_{N}(t) \omega(\lambda t) d t\right| \leq \int_{a}^{b}\left|R_{N}(t)\right| \omega(\lambda t) d t,
$$

and by Lemma 5.1, we get

$$
\left|\int_{a}^{b} R_{N}(t) \omega(\lambda t) d t\right| \leq\left|R_{N}(c)\right| \int_{a}^{b} \omega(\lambda t) d t .
$$

Thus

$$
\left|R_{N}(c)\right| \leq \frac{\int_{a}^{b}\left|R_{N}(t)\right| \omega(\lambda t) d t}{\int_{a}^{b} \omega(\lambda t) d t}=\hat{R}_{N}
$$

where $\hat{R}_{N}$ prescribes the upper bound error based on residual function with respect to the computation limit $N$ and $\omega(\lambda t)$. Thus, this completes the proof.

\section{Numerical problems}

In this section, we apply our method to solve stiff nonlinear problems, some of which are model problems in several applied sciences. To do this, we devise an efficient 


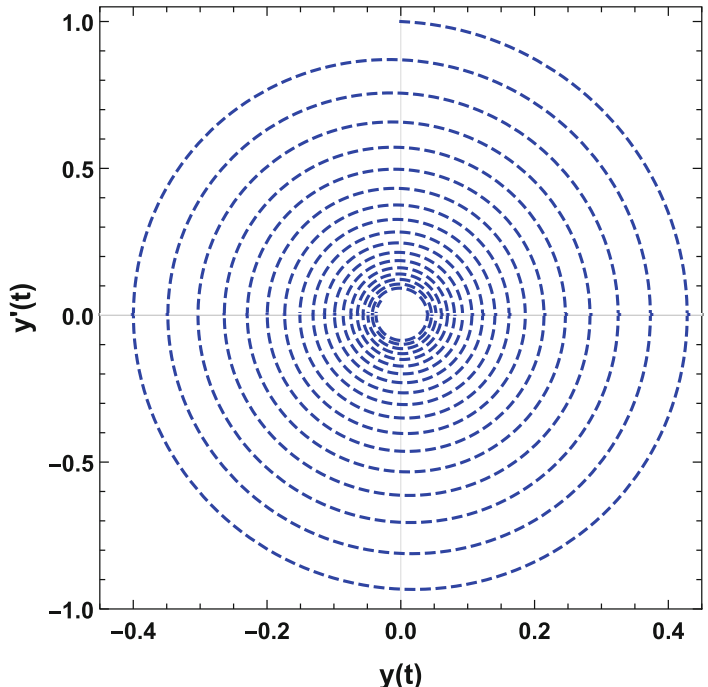

(a) Solution by Mathematica; $\alpha=2$.

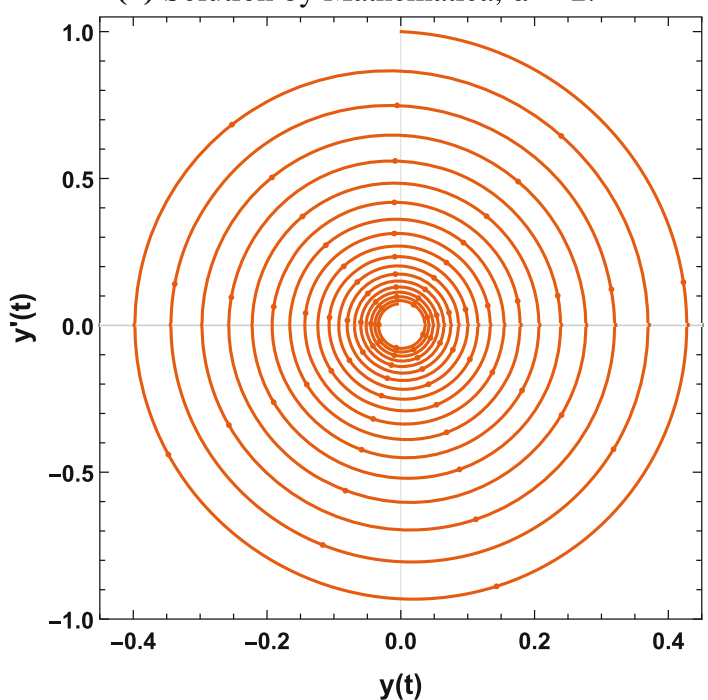

(b) $y_{7, P}(t) ; \alpha=2$.

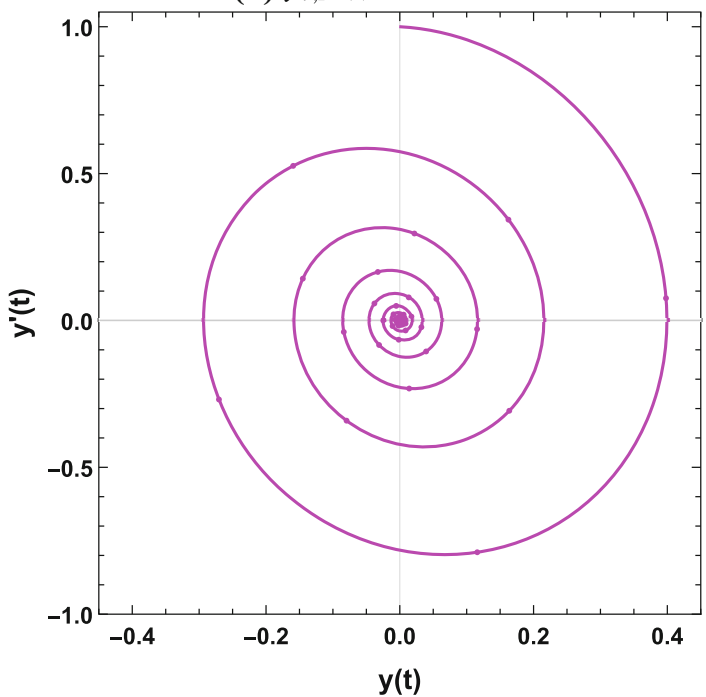

(c) $y_{7, P}(t) ; \alpha=1.9$
4Figure 6. Phase plane behaviour of the solutions for Problem 6.1 with low damping effect $\mu=0.1$ and $T=50$.

computer program on PC equipped with 8 GB RAM, 3.30 $\mathrm{GHz} \mathrm{CPU}$ and Mathematica 11. Hence, graphical and numerical results of high accuracy are obtained and demonstrated in figures and tables, respectively.

Problem 6.1 [44] Consider the fractional-order damped and unforced Duffing equation used in mechanical systems, chaos and theory of vibration:

Table 1. Comparison of the upper bound error $\hat{R}_{N}$ with respect to $\omega(\lambda t), N$ and $\alpha=2$ for Problem 6.1.

\begin{tabular}{lccccc}
\hline$\downarrow \omega(\lambda t) / N \rightarrow$ & 4 & 5 & 6 & 7 & 8 \\
\hline$\Gamma(t+\lambda)$ & & & & & \\
$\lambda=10^{-3}$ & $2.53 e-02$ & $7.58 e-02$ & $1.24 e-02$ & $8.27 e-03$ & $3.47 e-03$ \\
$\lambda=10^{-7}$ & $3.04 e-02$ & $6.54 e-02$ & $1.41 e-02$ & $9.57 e-03$ & $2.92 e-03$ \\
$\exp (\lambda t)$ & & & & & \\
$\lambda=1$ & $2.89 e-02$ & $1.76 e-01$ & $1.57 e-02$ & $1.20 e-02$ & $7.49 e-03$ \\
$\lambda=-1$ & $2.69 e-02$ & $1.25 e-01$ & $1.40 e-02$ & $9.73 e-03$ & $6.41 e-03$ \\
$\lambda=-10^{2}$ & $2.78 e-02$ & $5.75 e-02$ & $1.31 e-02$ & $9.35 e-03$ & $1.83 e-03$ \\
\hline
\end{tabular}

Table 2. Comparison of the upper bound error $\hat{R}_{N}$ with respect to $\omega(\lambda t), N$ and $\alpha=1.99$ for Problem 6.1.

\begin{tabular}{lcccc}
\hline$\downarrow \omega(\lambda t) / N \rightarrow$ & 5 & 6 & 7 & 8 \\
\hline$\Gamma(t+\lambda)$ & & & & \\
$\lambda=10^{-3}$ & $3.72 e-01$ & $4.07 e-02$ & $5.54 e-02$ & $1.62 e-02$ \\
$\lambda=10^{-1}$ & $2.55 e-01$ & $1.94 e-02$ & $4.28 e-02$ & $1.08 e-02$ \\
$\exp (\lambda t)$ & & & & \\
$\lambda=-1$ & $2.07 e-01$ & $1.31 e-02$ & $3.88 e-02$ & $1.08 e-02$ \\
\hline
\end{tabular}

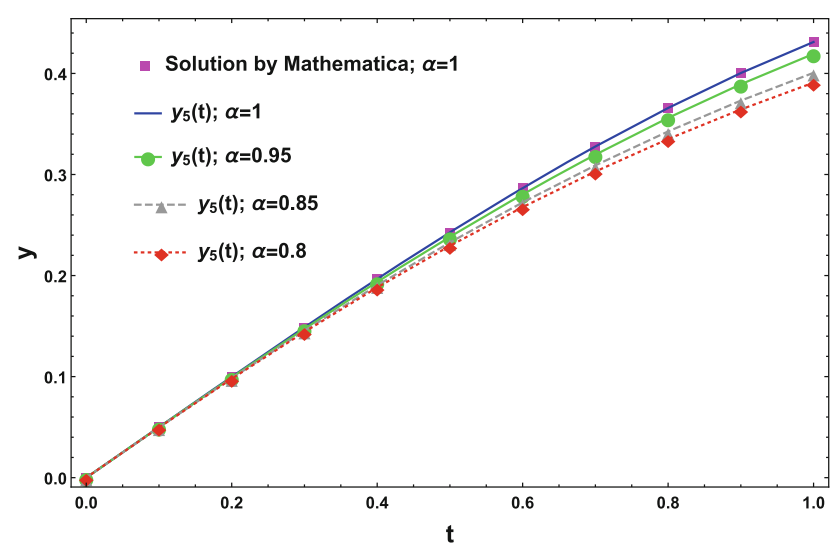

Figure 7. The effect of fractional derivative on the matching polynomial solution for Problem 6.2. 
Table 3. Comparison of the numerical results with respect to different methods for Problem 6.2 with $\alpha=1$.

\begin{tabular}{|c|c|c|c|c|}
\hline$t_{i}$ & $y_{5}\left(t_{i}\right)$ & $\begin{array}{c}\text { Runge-Kutta } \\
{[45]}\end{array}$ & $\begin{array}{c}\text { Chebyshev } \\
{[45]}\end{array}$ & $\begin{array}{c}\text { Haar wavelet } \\
{[46]}\end{array}$ \\
\hline 0.1 & 0.05004 & 0.05004 & 0.05004 & 0.05004 \\
\hline 0.2 & 0.09983 & 0.09983 & 0.09982 & 0.09983 \\
\hline 0.3 & 0.14887 & 0.14886 & 0.14885 & 0.14886 \\
\hline 0.4 & 0.19666 & 0.19965 & 0.19664 & 0.19665 \\
\hline 0.5 & 0.24270 & 0.24270 & 0.24275 & 0.24270 \\
\hline 0.6 & 0.28653 & 0.28653 & 0.28653 & 0.28653 \\
\hline 0.7 & 0.32771 & 0.32770 & 0.32771 & 0.32770 \\
\hline 0.8 & 0.36579 & 0.36577 & 0.36576 & 0.36577 \\
\hline 0.9 & 0.40039 & 0.40034 & 0.40040 & 0.40034 \\
\hline 1.0 & 0.43115 & 0.43105 & 0.43104 & 0.43105 \\
\hline
\end{tabular}

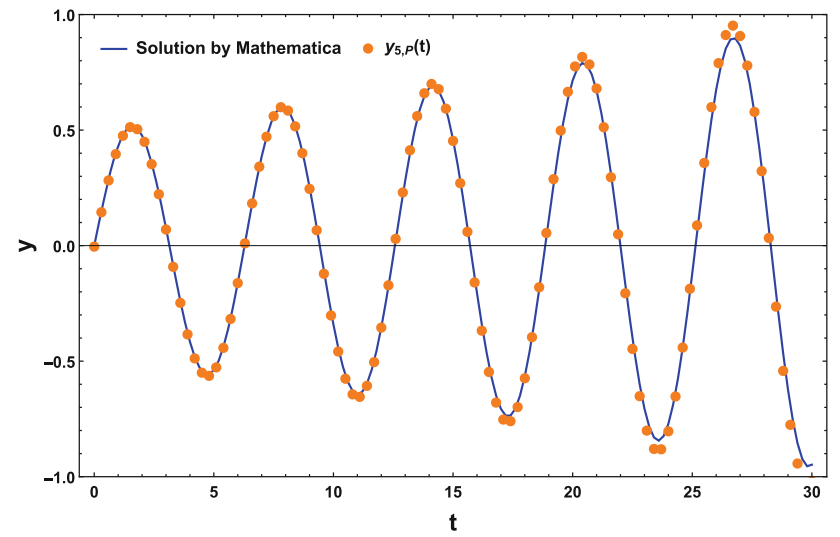

Figure 8. Oscillatory behaviour of the solutions for Problem 6.2 with $\alpha=1$ and $T=30$.

$$
y^{(\alpha)}(t)+\mu y^{\prime}(t)+5 y(t)+y^{3}(t)=0, t \in[0, T], \quad 1<\alpha \leq 2
$$

subject to the initial conditions $y(0)=0$ and $y^{\prime}(0)=1$. Here, the exact solution of the problem is unknown and $\mu$ is a damping effect. By the matrix equation (10), we can form the fundamental matrix equation of this problem as

$$
\begin{aligned}
& \left(\boldsymbol{P}_{0} \boldsymbol{X}+\boldsymbol{P}_{1} \boldsymbol{X}^{(1)}\right. \\
& \left.\quad+\boldsymbol{P}_{\alpha} \boldsymbol{X}^{(\alpha)}\right) \boldsymbol{K} \boldsymbol{Y}+\boldsymbol{Q}_{0,0,0} \boldsymbol{X} \boldsymbol{K} \overline{\boldsymbol{X}} \overline{\boldsymbol{K}} \overline{\overline{\boldsymbol{X}}} \overline{\overline{\boldsymbol{K}}} \overline{\overline{\boldsymbol{Y}}}=\boldsymbol{G} .
\end{aligned}
$$

Following the procedure described in section 3 and applying the Laplace-Padé method in section 4 we get the matching polynomial solutions in terms of $\mu, T$ and $\alpha$. Figure 2 clearly elucidates the behaviour of the matching polynomial solution along with the solution approached by Mathematica as $(\alpha=1)$

NDSolve $\left[\left\{y^{\prime \prime}[t]+\mu y^{\prime}[t]+5 y[t]+(y[t]) \hat{3}==0, y[0]==0\right.\right.$,

$$
\left.\left.y^{\prime}[0]==1\right\}, y[t],\{t, 0, T\}\right][[1,1,2]] \text {. }
$$

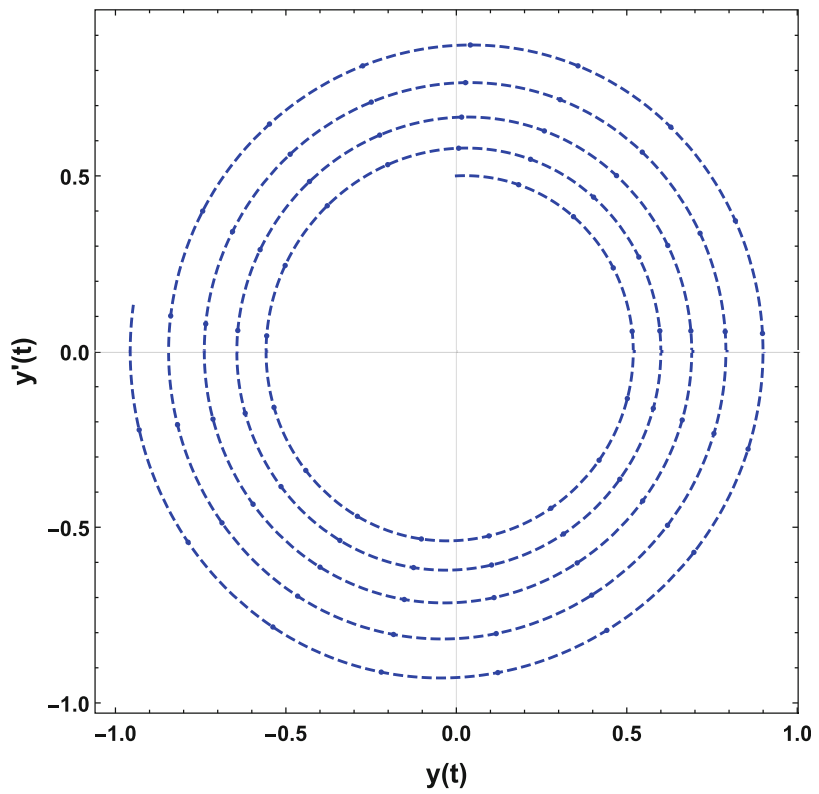

(a) Solution by Mathematica.

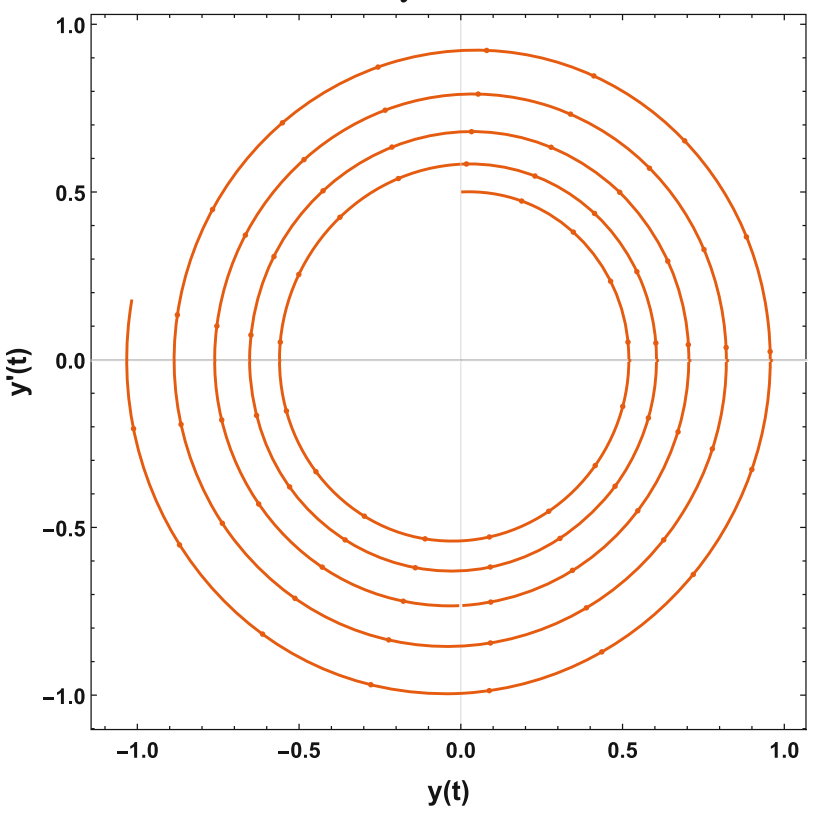

(b) $y_{5, P}(t)$.

Figure 9. Phase plane consistency of the solutions for Problem 6.2 with $\alpha=1$ and $T=30$.

Figures 3 and 4 illustrate the oscillatory behaviour of the solutions on the time interval $[0,50]$ and the effects of the fractional derivative and damping parameter on the matching polynomial solutions are clearly observed there. Figures 5 and 6 are also illustrate the phase plane consistency between the matching polynomial solution and the solution by Mathematica. The upper bound errors of the method with respect to the parameterized weight function $\omega(\lambda t), N$ and $\alpha$ are tabulated in tables 1 and 2. 


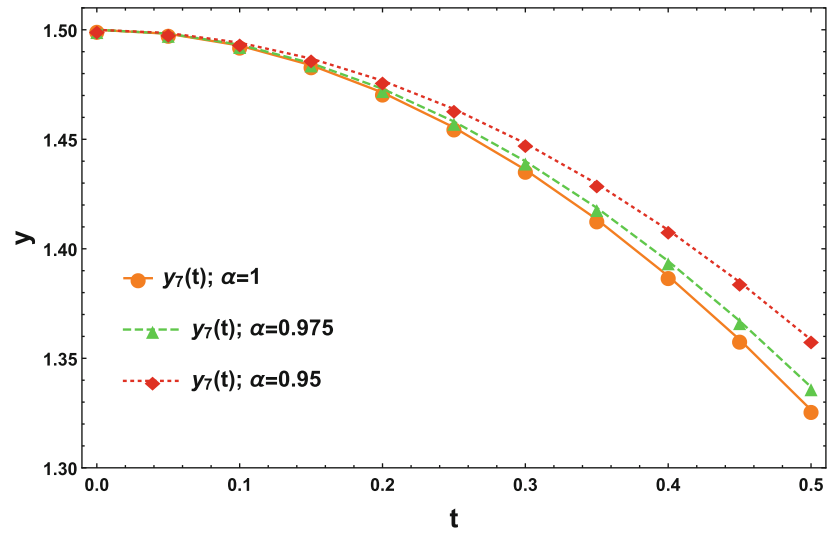

Figure 10. The effect of fractional derivative on the matching polynomial solution for Problem 6.3.

Problem 6.2 Consider the fractional-order unforced Van der Pol equation governing electrical circuits and chaotic systems:

$$
y^{(2 \alpha)}(t)-0.05\left(1-y^{2}(t)\right) y^{(\alpha)}(t)+y(t)=0,
$$

subject to the initial conditions $y(0)=0$ and $y^{\prime}(0)=0.5$. Here, $t \in[0, T], 0<\alpha \leq 1$ and the exact solution of the problem is unknown. Similarly, we solve the problem by employing $N=5$ and the Laplace-Padé method. In addition, Mathematica approaches this equation $(\alpha=1)$ in the following module:

$$
\begin{aligned}
& \text { NDSolve }\left[\left\{y^{\prime \prime}[t]-0.05 y^{\prime}[t]+0.05 y^{\prime}[t](y[t]) \hat{3}+y[t]\right.\right. \\
& \begin{array}{c}
=0, y[0]==0 \\
\left.\left.y^{\prime}[0]==0.5\right\}, y[t],\{t, 0, T\}\right][[1,1,2]] .
\end{array}
\end{aligned}
$$

We demonstrate the matching polynomial solution with respect to $\alpha$ in figure 7 . The authors in $[45,46]$ have obtained numerical solutions of this problem of integerorder type by employing Runge-Kutta [45], Chebyshev [45] and Haar wavelet methods [46]. In table 3, we make comparison between the present results and those by the mentioned methods. We observe that the present results coincide well with those of the mentioned methods. The matching polynomial solution and the solution by Mathematica are plotted in figure 8, and their phase plane behaviours are simulated in figure 9 .

Problem 6.3 Consider the fractional-order nonlinear differential equation proposed for a mechanical problem

$y^{(2 \alpha)}(t)-y(t)+\mu y^{2}(t) y^{(2 \alpha)}(t)+\mu y(t)\left(y^{(\alpha)}(t)\right)^{2}+y^{3}(t)=0$,

subject to the initial conditions $y(0)=A$ and $y^{\prime}(0)=0$. Here, $t \in[0,0.5], 0<\alpha \leq 1$. Lev et al [47] have recently established this mechanical problem modelling the velocity-dependent elastic module for $\alpha=1$. Wu and He [48] have employed the homotopy perturbation method to solve this problem with $\alpha=1$ and they have determined $A>2 / \sqrt{3} \cong 1.1547$ for its periodic solution. We here construct and solve a fractional-order form of this equation. For $N=7$, we obtain the solutions for $A=1.5$ and different $\alpha$. These are of the consistent form as seen in figure 10 .

Problem 6.4 [16] Consider the fractional-order nonlinear boundary value problem

$$
y^{(\alpha)}(t)+e^{-2 \pi} y^{2}(t)=g(t), t \in[0,1], 1<\alpha \leq 2,
$$

subject to the boundary conditions $y(0)=0$ and $y(1)=1$. Here, the exact solution is $y(t)=t^{7 / 2}$ and $g(t)=105 / 32 t^{2}+e^{2 \pi} t^{7}$ for $\alpha=1.5$. Employing the present method for different $N$ and $\alpha$, we easily get the solutions. Considering $\alpha$, table 4 compares the absolute errors that are obtained by the present method $(N=10)$ and LWM $(\hat{m}=12)[16]$, where the computation limit $N$ of the present method corresponds to $N=\hat{m}-1$. It is easily observed that our results are far better than

\begin{tabular}{|c|c|c|c|c|c|c|}
\hline$t_{i}$ & Ours $\alpha=1.1$ & $\alpha=1.1[16]$ & Ours $\alpha=1.3$ & $\alpha=1.3[16]$ & Ours $\alpha=1.5$ & $\alpha=1.5[16]$ \\
\hline 0.1 & $1.76 e-05$ & $2.91 e-04$ & $2.86 e-05$ & $1.98 e-04$ & $2.67 e-05$ & $9.70 e-05$ \\
\hline 0.2 & $3.49 e-05$ & $5.42 e-03$ & $1.68 e-05$ & $2.22 e-03$ & $3.35 e-05$ & $9.39 e-04$ \\
\hline 0.3 & $1.09 e-04$ & $6.02 e-03$ & $2.09 e-05$ & $3.02 e-03$ & $2.07 e-05$ & $1.51 e-03$ \\
\hline 0.4 & $1.57 e-04$ & $1.39 e-03$ & $5.83 e-05$ & $6.88 e-04$ & $3.96 e-07$ & $3.40 e-04$ \\
\hline 0.5 & $1.50 e-04$ & $8.41 e-03$ & $7.42 e-05$ & $4.51 e-03$ & $1.71 e-05$ & $2.42 e-03$ \\
\hline 0.6 & $8.19 e-05$ & $1.27 e-03$ & $5.77 e-05$ & $6.54 e-04$ & $2.03 e-05$ & $3.10 e-04$ \\
\hline 0.7 & $2.97 e-05$ & $5.77 e-03$ & $1.01 e-05$ & $3.03 e-03$ & $6.12 e-06$ & $1.48 e-03$ \\
\hline 0.8 & $1.54 e-04$ & $5.11 e-03$ & $5.79 e-05$ & $2.34 e-03$ & $2.41 e-05$ & $6.34 e-04$ \\
\hline 0.9 & $2.33 e-04$ & $3.43 e-03$ & $1.18 e-04$ & $4.02 e-03$ & $5.90 e-05$ & $4.67 e-03$ \\
\hline
\end{tabular}
those of LWM [16]. In addition, the upper bound errors of the method with respect to the parameterized weight function $\omega(\lambda t), N$ and $\alpha$ are given in table 5 .

Table 4. Comparison of the present absolute errors with those by LWM [16] for Problem 6.4 with different $\alpha$. 
Table 5. Comparison of the upper bound error $\hat{R}_{N}$ with respect to $\omega(\lambda t), N$ and $\alpha=1.5$ for Problem 6.4.

\begin{tabular}{ccccccc}
\hline$\downarrow \omega(\lambda t) / N \rightarrow$ & 4 & 5 & 6 & 7 & 8 & 9 \\
\hline$\Gamma(t+\lambda)$ & & & & & & 10 \\
$\quad \lambda=10^{-3}$ & $8.07 e-02$ & $3.32 e-02$ & $2.81 e-03$ & $2.25 e-03$ & $2.18 e-03$ & $1.25 e-03$ \\
$\quad \lambda=10^{-7}$ & $3.73 e-02$ & $1.56 e-02$ & $1.41 e-03$ & $1.17 e-03$ & $1.10 e-03$ & $6.25 e-04$ \\
$\exp (\lambda t)$ & & & & & $4.85 e-04$ \\
$\lambda=-1$ & $1.69 e-02$ & $7.10 e-02$ & $4.69 e-03$ & $2.73 e-03$ & $3.85 e-03$ & $1.59 e-03$ \\
$\lambda=-10^{3}$ & $1.70 e-02$ & $8.05 e-03$ & $1.20 e-03$ & $1.17 e-03$ & $9.52 e-04$ & $5.20 e-04$ \\
\hline
\end{tabular}

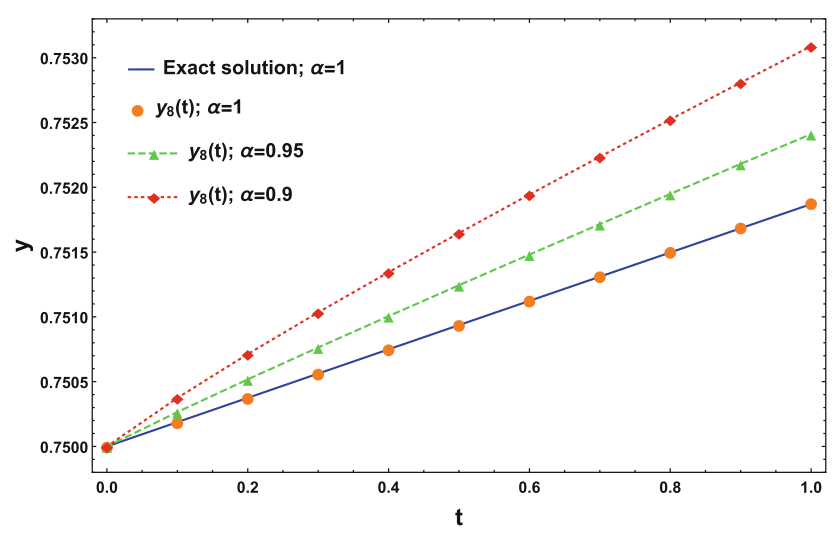

Figure 11. The effect of fractional derivative on the matching polynomial solution for Problem 6.5.

Problem 6.5 [10] Consider the fractional-order nonlinear logistic differential equation employed in social and biological sciences

$$
y^{(\alpha)}(t)-(0.01)^{\alpha} y(t)(1-y(t))=0, t \in[0,1], 0<\alpha \leq 1,
$$

subject to the initial condition $y(0)=0.75$. Here, the exact solution is $y(t)=0.75 /\left(0.75+(1-0.75) e^{-0.01 t}\right)$ for $\alpha=1$. We solve the problem by determining a low computation limit $N=8$. It is worth mentioning from figure 11 that the matching polynomial solution with respect to $\alpha$ is in a good agreement with the exact solution.

\section{Conclusions}

A matrix-collocation method based on the matching polynomial, a unified matrix expansion of fractional-order derivatives and a general matrix relation with cubic nonlinearity have been developed to solve stiff FDEs having cubic nonlinearity. Convergence analysis and error estimation have been established according to the second mean value theorem for integrals. Thus, we have clearly scrutinized the convergence of the method with respect to $N$, though the exact solutions of the problems are unknown. In addition, the prominence of the parameterized weight function $\omega(t)$ in our analysis has been observed in tables 1,2 and 5. Actually, this weight function has been used as the correction factor there. We want to point out from the figures that the present method completely describes the physical behaviour of the problems defined on the time intervals. Thereby, it can be easily expressed that the present method is reliable, coherent and accurate.

\section{Acknowledgements}

The authors would like to thank the reviewers for their constructive and valuable comments to improve the paper.

\section{References}

[1] Caputo M 1969 Elasticità e dissipazione. Bologna: Zanichelli

[2] Diethelm K 2010 The analysis of fractional differential equations. Berlin-Heidelberg: Springer-Verlag

[3] Engheta N 1996 On fractional calculus and fractional multipoles in electromagnetism. IEEE Trans. Antennas Propag. 44: 554-566

[4] Gülsu M, Öztürk Y and Anapalı A 2013 A collocation method for solving fractional Riccati differential equation. Adv. Appl. Math. Mech. 5: 872-884

[5] Hilfer R 2000 Applications of fractional calculus in physics. Singapore: World Scientific

[6] Kulish V V and Lage J L 2002 Application of fractional calculus to fluid mechanics. J. Fluids Eng. 124: 803-806

[7] Ortigueira M D, Ionescu C M, Machado J T and Trujillo J J 2015 Fractional signal processing and applications. Signal Process. 107: 197

[8] Podlubny I 1999 Fractional differential equations. New York: Academic Press

[9] Verotta D 2010 Fractional dynamics pharmacokineticspharmacodynamic models. J. Pharmacokinet. Pharmacodyn. 37: 257-276

[10] West B J 2015 Exact solution to fractional logistic equation. Physica A 429: 103-108

[11] Kürkçü Ö K, Aslan E and Sezer M 2019 A novel graphoperational matrix method for solving multidelay fractional differential equations with variable coefficients and a 
numerical comparative survey of fractional derivative types. Turk. J. Math. 43: 373-392

[12] Kürkçü Ö K, Aslan E and Sezer M 2019 An integrated numerical method with error analysis for solving fractional differential equations of quintic nonlinear type arising in applied sciences. Math. Methods Appl. Sci.https://doi.org/10. 1002/mma.5708

[13] Yüzbaşı Ş 2013 Numerical solutions of fractional Riccati type differential equations by means of the Bernstein polynomials. Appl. Math. Comput. 219: 6328-6343

[14] Saeed U 2017 CAS Picard method for fractional nonlinear differential equation. Appl. Math. Comput. 307: 102-112

[15] Yarmohammadi M, Javadi S and Babolian E 2018 Spectral iterative method and convergence analysis for solving nonlinear fractional differential equation. J. Comput. Phys. 359: $436-450$

[16] Rehman M and Khan R A 2011 The Legendre wavelet method for solving fractional differential equations. Commun. Nonlinear Sci. Numer. Simul. 16: 4163-4173

[17] Baykuş Savaşaneril N and Sezer M 2017 Hybrid TaylorLucas collocation method for numerical solution of highorder pantograph type delay differential equations with variables delays. Appl. Math. Inf. Sci. 11: 1795-1801

[18] Baykuş Savaşaneril N and Sezer M 2011 Solution of high-order linear Fredholm integro-differential equations with piecewise intervals. Numer. Methods Partial Differ. Equ. 27: 1327-1339

[19] Kurt N and Sezer M 2008 Polynomial solution of high-order linear Fredholm integro differential equations with constant coefficients. J. Franklin Inst. 345: 839-850

[20] Kürkçü Ö K, Aslan E and Sezer M 2017 A numerical method for solving some model problems arising in science and convergence analysis based on residual function. Appl. Numer. Math. 121: 134-148

[21] Baleanu D, Mousalou A and Rezapour S 2017 A new method for investigating approximate solutions of some fractional integro-differential equations involving the Caputo-Fabrizio derivative. Adv. Differ. Equ. 2017

[22] Caputo M and Fabrizio M 2015 A new definition of fractional derivative without singular kernel. Prog. Fract. Differ. Appl. 1: 1-13

[23] Losada $\mathbf{J}$ and Nieto $\mathbf{J} \mathbf{J} 2015$ Properties of a new fractional derivative without singular kernel. Prog. Fract. Differ. Appl. 1: 87-92

[24] Atangana A and Baleanu D 2016 New fractional derivatives with nonlocal and non-singular kernel: theory and application to heat transfer model. Therm. Sci. 20: 763-769

[25] Doungmo Goufo E F 2016 Application of the Caputo-Fabrizio fractional derivative without singular kernel to Korteweg-de Vries-Bergers equation. Math. Model. Anal. 21: 188-198

[26] Doungmo Goufo E F 2016 Chaotic processes using the twoparameter derivative with non-singular and non-local kernel: basic theory and applications. Chaos Interdiscip. J. Nonlinear Sci. 26: 084305

[27] Heilmann O J and Lieb E H 1972 Theory of monomer-dimer systems. Commun. Math. Phys. 25: 190-232.
[28] Farrell E J 1979 An introduction to matching polynomials. $J$. Comb. Theory Ser. B 27: 75-86

[29] Gutman I 1977 The acyclic polynomial of a graph. Publ. Inst. Math. Beograd 22: 63-69.

[30] Aihara J 1979 Matrix representation of an olefinic reference structure for monocyclic conjugated compounds. Bull. Chem. Soc. Japan 52: 1529-1530

[31] Harary F 1969 Graph theory. New York: Addison-Wesley

[32] Gutman I 1979 The matching polynomial. MATCH Commun. Math. Comput. Chem. 6: 75-91

[33] Godsil C D and Gutman I 1981 On the theory of the matching polynomial. J. Graph Theor. 5: 137-145

[34] Weisstein E W Matching polynomial. In: Math World: A Wolfram Web Resource. http://mathworld.wolfram.com/ MatchingPolynomial.html

[35] Hosoya H 1988 On some counting polynomials in chemistry. Discrete Appl. Math. 19: 239-257

[36] Ghosh T, Mondal S and Mandal B 2018 Matching polynomial coefficients and the Hosoya indices of poly(pphenylene) graphs. Mol. Phys. 116: 361-377

[37] Araujo O, Estrada M, Morales D A and Rada J 2005 The higher-order matching polynomial of a graph. Int. J. Math. Math. Sci. 10: 1565-1576

[38] Aslan E 2014 A measure of graphs vulnerability: edge scattering number. Bull. Int. Math. Virtual Inst. 4: 53-60

[39] Bacak-Turan G and Kırlangıç A 2011 Neighbor rupture degree and the relations between other parameters. Ars Comb. 102: 333-352

[40] Momani S and Ertürk V S 2008 Solutions of non-linear oscillators by the modified differential transform method. Comput. Math. Appl. 55: 833-842

[41] Sweilam N H and Khader M M 2009 Exact solutions of some coupled nonlinear partial differential equations using the homotopy perturbation method. Comput. Math. Appl. 58: 2134-2141

[42] Hobson E W 1909 On the second mean value theorem of the integral calculus. Proc. London Math. Soc. 2-7: 14-23 doi: https://doi.org/10.1112/plms/s2-7.1.14

[43] Dixon A 1929 The second mean value theorem in the integral calculus. Math. Proc. Cambridge 25: 282-284

[44] Nourazar S and Mirzabeigy A 2013 Approximate solution for nonlinear Duffing oscillator with damping effect using the modified differential transform method. Sci. Iran. B 20: 364-368

[45] Akyüz-Daşcıoğlu A and Çerdik-Yaslan H 2011 The solution of high-order nonlinear ordinary differential equations by Chebyshev series. Appl. Math. Comput. 217: 5658-5666

[46] Kaur H, Mittal R C and Mishra V 2014 Haar wavelet solutions of nonlinear oscillator equations. Appl. Math. Model. 38: 4958-4971

[47] Lev B I, Tymchyshyn V B and Zagorodny A G 2017 On certain properties of nonlinear oscillator. Phys. Lett. A 381: 3417-3423

[48] Wu Y and He J H 2018 Homotopy perturbation method for nonlinear oscillators with coordinate-dependent mass. $R e$ sults Phys. 10: 270-271 